\title{
COVID19 infection in a patient undergoing treatment for Paroxysmal Nocturnal Hemoglobinuria (PNH) with Ravulizumab
}

\author{
Sufana Shikdar ${ }^{1,3^{*}}$, Azra Borogovac ${ }^{1}$, Elabdallah Mohamad² and Mohamad Khawandanah
}

\begin{abstract}
Background: In the recent COVID19 pandemic, patients with hematological disorders were considered at high risk for severe disease. Limited data is available regarding the course of COVID19 infection in this subgroup.

Case Presentation: We describe a case of a 32-year-old man with paroxysmal nocturnal hemoglobinuria (PNH) undergoing treatment with ravulizumab (Ultomiris) who presented with COVID19 infection. He experienced only mild symptoms and had a rapid recovery from COVID19 infection.

Conclusion: This case may demonstrate the beneficial effects of ravulizumab on complement mediated inflammatory damage linked with COVID19 infection especially in PNH patients.
\end{abstract}

Keywords: COVID19, Paroxysmal nocturnal hemoglobinuria, Complement inhibitor

\section{Background}

Coronavirus disease 2019 (COVID-19) has become a global pandemic with at least 229.3 million confirmed cases and a total of 4,700,000 deaths worldwide as of September 2021 [1]. In December 2019, an outbreak of COVID-19 disease was detected in China caused by the Severe Acute Respiratory Syndrome Coronavirus-2 (SARS-CoV-2). The clinical manifestation of COVID-19 is characterized by respiratory distress, and in more severe cases can progress towards acute respiratory distress syndrome (ARDS) and death [2]. COVID-19 infection carries a potentially fatal risk, especially with an immunocompromised state and multiple comorbidities [3]. Several mutations of SARS-CoV-2 have been

\footnotetext{
*Correspondence: Sufana.shikdar@gmail.com

'Department of Hematology/Oncology, Stephenson Cancer Center,

University of Oklahoma Health Sciences Center, 73104 Oklahoma City, United States, OK

${ }^{3}$ Hematology/Oncology fellow, Department of Medicine, Division of Hematology-Oncology Stephenson Cancer Center, University of Oklahoma Health Sciences Center, 800 NE 10th St., 6th Floor, OK 73104 Oklahoma City, United States

Full list of author information is available at the end of the article
}

reported so far, and most recently a new mutation in the Delta variant (B.1.617.2 lineage) was found which seems to be highly contagious and is now the dominant variant globally [4]. Meanwhile, on December 11, 2020, FDA approval of the Pfizer-BioNTech in individuals 16 years of age and older vaccine has opened a new door to fight against the global pandemic; currently, we have three FDA approved vaccines available in the US [4]. Even though the COVID vaccine is highly effective at preventing severe illness, they do not give full protection in preventing and transmitting the COVID-19 infection.

COVID-19 infection causes hyperactivation of the complement system and excessive inflammatory response leading to worsening lung injury and poor clinical outcomes $[2,5]$. The interaction between the complement system and COVID-19 infection raises the possibility that immunosuppression could be a promising approach to inhibit the consequences of complement mediated inflammatory destruction in COVID-19 infection. Moreover, patients with $\mathrm{PNH}$ may be vulnerable to COVID-19 complications due to impaired immune status [6]. Based on this immunological rationale, it was 
speculated that the therapeutic use of complement inhibitors might be an effective strategy to control systemic inflammation in COVID-19 infection.

Although complement blockade strategies are being prospectively studied in clinical trials and reported in case reports, no cases of PNH with COVID-19 infection managed with complement blocker agents have been reported $[7,8]$. Herein, we report the first case of COVID19 pneumonia in a patient with $\mathrm{PNH}$ on treatment with intravenous ravulizumab, a complement component C5 inhibitor. He had a favorable clinical course linked to COVID19 infection and was discharged without any complications.

\section{Case presentation}

Our patient was a 32-year-old Nigerian immigrant male who presented around September 2018 with a clinical picture of severe aplastic anemia. The patient received one cycle of Horse antithymocyte globulin (ATG; $40 \mathrm{mg} / \mathrm{kg}$ once daily for 4 days); two weeks of prednisone (100 mg orally twice a day, then tapered), and six months of eltrombopag (150 mg orally daily) and cyclosporin A $(10 \mathrm{mg} / \mathrm{kg}$ orally daily). The diagnosis of combined $\mathrm{PNH}$ and aplastic anemia due to bone marrow failure, hemolysis, and detection of $\mathrm{PNH}$ clone in addition to aplastic hypocellular marrow was made, and the patient was started with eculizumab for 5 cycles, then switched to ravulizumab around March 2019 with the last dose in February 2020. He remained profoundly pancytopenic and he was waiting for an allogeneic transplant.

He presented in March 2020 with fever, runny nose, dry cough, and altered taste (dysgeusia) for 4 days. Physical examination revealed normal blood pressure, fever $\left(38.3{ }^{\circ} \mathrm{C}\right)$, tachypnea (30 breaths per min) with baseline oxygen saturation of $95 \%$ in room air. The COVID19 test using targeted rich multiplex polymerase chain reaction of a nasopharyngeal swab came back positive for SARS-CoV-2 infection. A chest radiography did not show infiltrates (Fig. 1). Infectious workup including blood and urine cultures were negative. Respiratory viral panel was negative for influenza A and B. Laboratory tests revealed WBC 1.93 cells/mm3, absolute neutrophil count (ANC) 950 cells $/ \mathrm{mm} 3$, Hb $8 \mathrm{gm} / \mathrm{l}$, Platelet $37,000 / \mathrm{mm} 3$; increased levels of acute phase reactants, including CRP (108 mg/l; normal range <5 mg/l), ferritin (3355 ng/ $\mathrm{ml}$; normal range $10-322 \mathrm{ng} / \mathrm{ml}$ ), serum lactate dehydrogenase (364 U/l; normal range 112-236 U/l), and fibrinogen $(615 \mathrm{mg} / \mathrm{dl}$; normal value $150-450 \mathrm{mg} /$ dl). The patient was treated with azithromycin, hydroxychloroquine, and prophylactic enoxaparin. He required no oxygen, remained afebrile, and oxygen saturation was maintained around $95 \%$ in room air. $\mathrm{He}$ demonstrated a rapid and progressive improvement in his symptoms and was discharged from the

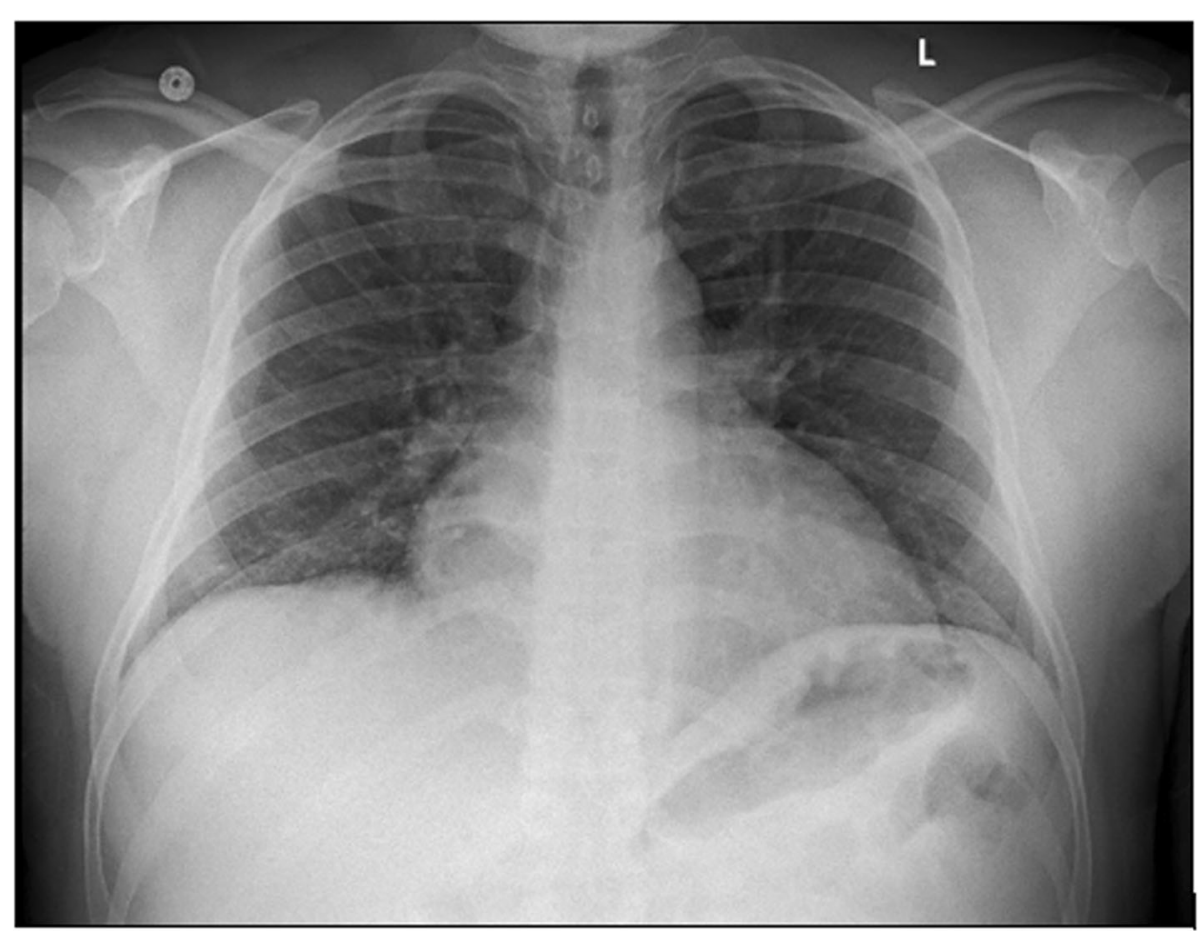

Fig. 1 Chest Xray on admission: No infiltrates noted with COVID19 infection 
hospital 4 days after diagnosis with no immediate complications and no evidence of breakthrough hemolysis.

\section{Discussion and conclusions}

$\mathrm{PNH}$ is a clonal disorder of hematopoietic progenitor cells caused by an acquired mutation of the X-linked phosphatidylinositol glycans class A (PIG-A) gene [9]. The absence of glycosylphosphatidylinositol (GPI) anchored complement regulatory proteins CD55 and CD59 from the membrane of circulatory cells is responsible for the activation of the complement system on the surface of the red cell membrane. This leads to complement mediated intravascular hemolysis, activation of platelets, and the coagulation cascade resulting in a hypercoagulable state [9]. PNH, although rare, can be fatal and includes an increased risk of thromboembolism and severe end-organ damage. Approximately, $35 \%$ of patients die within five years if untreated due to thrombosis and related complications [10].

There is a close relationship between $\mathrm{PNH}$ and aplastic anemia (AA) especially when PNH evolves into bone marrow hypoplasia, which is a hallmark of AA. An overlap entity can be the presenting symptom i.e., dual diagnosis. Three presentations lead to overlap or dual diagnosis: (1) aplastic anemia on original presentation then the discovery of PNH clone, (2) PNH presentation then evolution to aplastic anemia, and (3) Aplastic anemia and $\mathrm{PNH}$ dual diagnosis on presentation [11]. Our patient presented with the dual diagnosis of $\mathrm{PNH}$ manifested by hemolysis and jaundice in addition to profoundly aplastic bone marrow.

Ravulizumab (ALXN1210; Alexion Pharmaceuticals, Inc) is a second generation humanized monoclonal antibody that prevents complement protein 5 (C5) cleavage and activation, ultimately blocking membrane attack complex (MAC) formation in the complement pathway [12]. Ravulizumab is now approved by the FDA to treat PNH. It has an extended 8-week maintenance dosing interval and reduces the need for frequent drug administration with subsequent improvement in symptom control and better quality of life.

The complement system activation is a critical component in the sequelae of COVID19 infection. Evidence suggests that severe outcomes in COVID19 infection are attributed to the excessive activation of the complement cascade leading to acute lung injury and associated with an increased prothrombotic state $[2,5,13,14]$. Notably, C5a concentration was noted to be higher in patients with COVID19 infection $[2,14]$. Based on these observations, complement component blockers could be used as potential therapeutic targets in COVID-19 patients.
Several clinical trials have been ongoing to target complement mediated inflammatory response in the emerging COVID19 outbreak. An anti C3 agent, compstatin analog Cp40/AMY-101 has shown efficacy in complement mediated severe ARDS in COVID19 patients, and a phase II clinical trial is ongoing [7]. In addition, a multicenter phase II/III trial using a monoclonal neutralizing anti-C5a antibody (IFX-1) is recruiting patients with severe COVID19 pneumonia [8]. Preliminary PK/PD analysis of ALXN1210-COV-305 phase 3 trial found that patients with severe COVID-19 infection treated with modified ravulizumab dosing regimen resulted in improved clinical outcomes $[15,16]$. Alexion also initiated a clinical trial in critical COVID19 patients on a related drug, eculizumab (NCT04288713) [17]. A proof-of-concept study by Annane et al. found survival benefits with eculizumab in patients with severe COVID-19 [18]. Clinical and experimental research is ongoing to optimize therapeutic interventions targeting the complement system [19-22]. For example, zilucoplan, an anti-C5 drug, is being evaluated in a phase 2 ACCORD trial for hospitalized COVID-19 patients [19]. Also, the TACTIC-R trial is currently assessing the efficacy of ravulizumab among Pre-ICU COVID-19 patients [20]. In addition, Multi-center Pilot Trial (PROTECT-COVID-19) is evaluating the efficacy of conestat alpha, the $\mathrm{C} 1$ esterase inhibitor in severe COVID-19 infection [21], and I-SPY COVID-19 trial is investigating the benefit of anti-MASP-2 antibody Narsoplimab in critically ill COVID-19 patients [22]. While these trials posit a new potential treatment strategy, the therapeutic use of complement inhibitors in COVID-19 immunocompromised patients is limited. Few case reports have shown a promising effect of complement inhibitors in COVID-19 patients. Eculizumab used in the patients with thrombotic microangiopathic anemia in the setting of COVID19 showed dramatic improvement in kidney function [23]. Another patient with a transplanted kidney who was on Eculizumab for the atypical hemolytic uremic syndrome (HUS), was treated for COVID19 infection and had a full recovery [24]. Similarly, a patient with $\mathrm{PNH}$ diagnosed with COVID-19 pneumonia showed fast recovery with the initiation of eculizumab [25].

Immunocompromised patients are at higher risk for severe COVID19 infections and may experience prolonged hospitalization due to COVID19-related morbidity, and mortality. Additionally, administration of most scheduled anti-cancer treatments may be delayed or interrupted. In our case, the continuation of ravulizumab during COVID19 infection may have assisted a prompt recovery by attenuating complement activation.

The CDC recommends adopting strategies to minimize the risk of COVID19 exposure, such as limiting contacts between patients and healthcare providers. Ravulizumab has a longer therapeutic window and therefore a less 
frequent dosing schedule than eculizumab. We recommend against interrupting or holding the dose of ravulizumab even during systemic inflammation to combat complement mediated tissue damage and multiorgan failure. We also recommend switching the every 8-weeks dosing schedule of eculizumab with ravulizumab to minimize the exposure to COVID19 and other infections and reduce the economic burden on hospital systems due to less frequent dosing.

Given the worldwide COVID19 pandemic, specific clinical and economically cost-effective therapeutic options should be explored and prioritized. Treatment with ravulizumab during COVID19 pneumonia was safe, cost-effective, and found to have a favorable clinical course in a patient with $\mathrm{PNH}$. A systemic prospective trial is warranted to demonstrate the utility and usefulness of ravulizumab in patients with $\mathrm{PNH}$ in the COVID-19 outbreak.

\section{Acknowledgements}

None.

\section{Authors' contributions}

SS made substantial contributions to the conception and design, drafting of the manuscript, analysis, and interpretation of data, and revising the manuscript and gave final approval for publication. $A B$, and $E M$, provided clinical care for the patient and critically revised the manuscript for important intellectual content. MK served as primary consultant in the management of the patient and made substantial contributions to the conception and design; analysis and interpretation of data; drafting of the manuscript; and revising the manuscript critically for important intellectual content; and gave final approval of the version to be published. All authors read and approved the final manuscript.

\section{Funding}

Not applicable.

\section{Availability of data and materials}

Not applicable.

\section{Declarations}

\section{Ethics approval and consent to participate}

Not applicable as no patient identifiable data was included.

\section{Consent for publication}

Informed consent for publication of their clinical details and Imaging was obtained from the patient.

\section{Competing interests}

The authors declare that they have no competing interests.

\section{Author details}

'Department of Hematology/Oncology, Stephenson Cancer Center, University of Oklahoma Health Sciences Center, 73104 Oklahoma City, United States, OK. ${ }^{2}$ Department of Internal Medicine, University of Oklahoma Medical Center (OUMC), 73104 Oklahoma City, United States, OK. ${ }^{3}$ Hematology/Oncology fellow, Department of Medicine, Division of Hematology-Oncology Stephenson Cancer Center, University of Oklahoma Health Sciences Center, 800 NE 10th St., 6th Floor, OK 73104 Oklahoma City, United States.
Received: 24 September 2020 Accepted: 11 October 2021

Published online: 21 October 2021

\section{References}

1. World Health Organization. Coronavirus [internet]. https:/www.who.int/da ta. Accessed 22 September 2021.

2. Bosmann M. Complement activation during critical illness: Current findings and an outlook in the era of COVID-19. Am J Respir Crit Care Med. 2020;202: 163-5.

3. Najjar S, Najjar A, Chong DJ, Pramanik BK, Kirsch C, Kuzniecky RI, et al. Central nervous system complications associated with SARS-CoV-2 infection: integrative concepts of pathophysiology and case reports. J Neuroinflammation. 2020:17:231.

4. Aleem A, AB AS, Slenker AK. Emerging Variants of SARS-CoV-2 And Novel Therapeutics Against Coronavirus (COVID-19).

5. Fletcher-Sandersjöö A, Bellander B-M. Is COVID-19 associated thrombosis caused by overactivation of the complement cascade? A literature review. Thromb Res. 2020;194:36-41.

6. Malard F, Genthon A, Brissot E, van de Wyngaert Z, Marjanovic Z, Ikhlef S, et al. COVID-19 outcomes in patients with hematologic disease. Bone Marrow Transplant [Internet]. 2020; Available from: https://doi.org/10.1038/ s41409-020-0931-4.

7. Mastaglio S, Ruggeri A, Risitano AM, Angelillo P, Yancopoulou D, Mastellos DC, et al. The first case of COVID-19 treated with the complement C3 inhibitor AMY-101. Clin Immunol. 2020;215:108450

8. Vlaar A.Open-label. Randomized Study of IFX-1 in Patients With Severe COVID-19 Pneumonia (PANAMO) 2020. [accessed 2020 July ]Available from: https://clinicaltrialsgov/ct2/show/NCT04333420 [Ref list].

9. Rosti V. The molecular basis of paroxysmal nocturnal hemoglobinuria. Haematologica. 2000;85:82-7.

10. Gembillo G, Siligato R, Cernaro V, Santoro D. Complement inhibition therapy and dialytic strategies in paroxysmal nocturnal hemoglobinuria: The nephrologist's opinion. J Clin Med. 2020;9:1261.

11. Olson TS. Aplastic anemia: Pathogenesis, clinical manifestations, and diagnosis. UpToDate. Accessed 22 September 2021 from https://www. uptodate.com/contents/aplastic-anemia-pathogenesis-clinical-manifesta tions-and diagnosis.

12. Stern RM, Connell NT. Ravulizumab: a novel C5 inhibitor for the treatment of paroxysmal nocturnal hemoglobinuria. Ther Adv Hematol. 2019;10: 2040620719874728

13. Porfidia A, Valeriani E, Pola R, Porreca E, Rutjes AWS, Di Nisio M. Venous thromboembolism in patients with COVID-19: Systematic review and metaanalysis. Thromb Res. 2020;196:67-74.

14. Risitano AM, Mastellos DC, Huber-Lang M, Yancopoulou D, Garlanda C, Ciceri F, et al. Complement as a target in COVID-19? Nat Rev Immunol. 2020;20:343-4.

15. Efficacy and Safety Study of IV Ravulizumab in Patients With COVID-19 Severe Pneumonia. - Full Text View - ClinicalTrials.Gov [Internet]. Clinicaltrials.gov. [cited 2020 Aug 29]. Available from: http://Clinicaltrials.gov/ ct2/show/NCT04369469.

16. McEneny-King AC, Monteleone JP, Kazani SD, Ortiz SR. Pharmacokinetic and pharmacodynamic evaluation of ravulizumab in adults with severe Coronavirus Disease 2019. Infectious Diseases Therapy. $2021 ; 10(2): 1045-54$.

17. Eculizumab. (Soliris) in covid-19 infected patients (SOLID-C19). Available at: https://linicaltrials.gov/ct2/show/NCT04288713. Published February 28, 2020. Updated March 30, 2020. Accessed 20 July 2020.

18. Annane $D$, Heming N, Grimaldi-Bensouda L, Frémeaux-Bacchi V, Vigan $M$, Roux AL, Marchal A, Michelon H, Rottman M, Moine P. Eculizumab as an emergency treatment for adult patients with severe COVID-19 in the intensive care unit: a proof-of-concept study. EClinicalMedicine. 2020 Nov 1; 28:100590.

19. Wilkinson T, Dixon R, Page C, Carroll M, Griffiths G, Ho LP, De Soyza A, Felton T, Lewis KE, Phekoo K, Chalmers JD. ACCORD: a multicentre, seamless, phase 2 adaptive randomisation platform study to assess the efficacy and safety of multiple candidate agents for the treatment of COVID-19 in hospitalised patients: a structured summary of a study protocol for a randomised controlled trial. Trials. $2020 ; 21(1): 1-3$

20. Kulkarni S, Fisk M, Kostapanos M, Banham-Hall E, Bond S, Hernan-Sancho E, Norton S, Cheriyan J, Cope A, Galloway J, Hall F. Repurposed immunomodulatory drugs for Covid-19 in pre-lCu patients-mulTi-Arm Therapeutic study in pre-ICu patients admitted with Covid-19-Repurposed 
Drugs (TACTIC-R): A structured summary of a study protocol for a randomised controlled trial. Trials. $2020 ; 21(1): 1-3$.

21. Urwyler P, Charitos P, Moser S, Heijnen IA, Trendelenburg M, Thoma R, Sumer J, Camacho-Ortiz A, Bacci MR, Huber LC, Stüssi-Helbling M. Recombinant human C1 esterase inhibitor (conestat alfa) in the prevention of severe SARSCoV-2 infection in hospitalized patients with COVID-19: A structured summary of a study protocol for a randomized, parallel-group, open-label, multi-center pilot trial (PROTECT-COVID-19). Trials. $2021 ; 22(1): 1-3$.

22. I-SPY, COVID-19. TRIAL: an adaptive platform trial for critically ill patients full text view. Available from: ClinicalTrials.gov. Accessed May23, 2021.

23. Boudhabhay I, Rabant M, Roumenina LT, Coupry LM, Poillerat V, Marchal A, Frémeaux-Bacchi V, El Karoui K, Monchi M, Pourcine F. Case Report: Adult Post-COVID-19 Multisystem Inflammatory Syndrome and Thrombotic Microangiopathy. Front Immunol. 2021;23:2284.

24. Mentese IB, Velioglu A, Aykent B, Gunay DS, Arikan H, Tuglular S. Course of COVID-19 Pneumonia in a Kidney Transplant Patient Receiving Eculizumab. Progress in Transplantation. Jun. 2021;18:15269248211024629.

25. Sokol J, Nehaj F, Mokan M, Lisa L, Stasko J. COVID19 infection in a patient with paroxysmal nocturnal hemoglobinuria: A case report. Medicine. 2021 May 21;100(20).

\section{Publisher's Note}

Springer Nature remains neutral with regard to jurisdictional claims in published maps and institutional affiliations.

Ready to submit your research? Choose BMC and benefit from:

- fast, convenient online submission

- thorough peer review by experienced researchers in your field

- rapid publication on acceptance

- support for research data, including large and complex data types

- gold Open Access which fosters wider collaboration and increased citations

- maximum visibility for your research: over $100 \mathrm{M}$ website views per year

At BMC, research is always in progress.

Learn more biomedcentral.com/submissions 